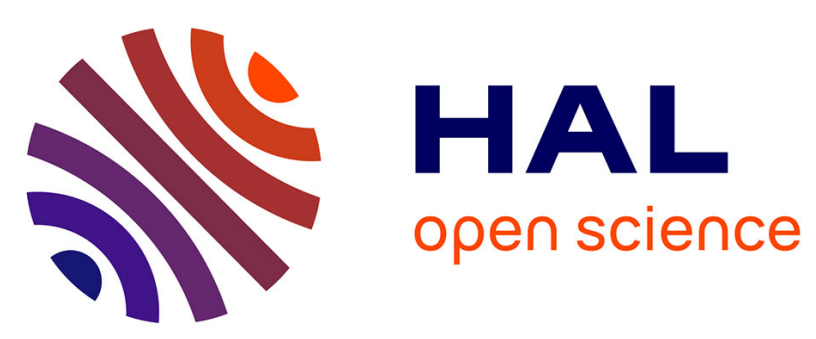

\title{
Thermally Activated Dissipation and Upper Critical Magnetic Field under the Strong Electrostatic Field in the BiPbSrCaCuO Thin Film
}

V. Lysenko, Y. Gomeniuk, I. Tyagulski, I. Osiyuk, V. Lozovski, V. Varyukhin

\section{- To cite this version:}

V. Lysenko, Y. Gomeniuk, I. Tyagulski, I. Osiyuk, V. Lozovski, et al.. Thermally Activated Dissipation and Upper Critical Magnetic Field under the Strong Electrostatic Field in the BiPbSrCaCuO Thin Film. Journal de Physique IV Proceedings, 1996, 06 (C3), pp.C3-271-C3-276. 10.1051/jp4:1996341 . jpa-00254259

HAL Id: jpa-00254259

https://hal.science/jpa-00254259

Submitted on 1 Jan 1996

HAL is a multi-disciplinary open access archive for the deposit and dissemination of scientific research documents, whether they are published or not. The documents may come from teaching and research institutions in France or abroad, or from public or private research centers.
L'archive ouverte pluridisciplinaire HAL, est destinée au dépôt et à la diffusion de documents scientifiques de niveau recherche, publiés ou non, émanant des établissements d'enseignement et de recherche français ou étrangers, des laboratoires publics ou privés. 


\title{
Thermally Activated Dissipation and Upper Critical Magnetic Field under the Strong Electrostatic Field in the BiPbSrCaCuO Thin Film
}

\author{
V.S. Lysenko, Y.V. Gomeniuk, I.P. Tyagulski, I.N. Osiyuk, V.Z. Lozovski and V.N. Varyukhin* \\ Institute of Semiconductor Physics, National Academy of Sciences of Ukraine, 45 Prosp. Nauki, \\ 252650 Kiev, Ukraine \\ * Donetsk Physico-Technical Institute, National Academy of Sciences of Ukraine, 72 ul.R. Luxemburg, \\ 340114 Donetsk, Ukraine
}

\begin{abstract}
Three different regimes of thermally activated dissipation behaviour were determined in the superconducting $(\mathrm{Bi}, \mathrm{Pb})_{2} \mathrm{Sr}_{2} \mathrm{Ca}_{2} \mathrm{Cu}_{3} \mathrm{O}_{\mathrm{X}}$ thin film in dependence on the external magnetic field. The negative electrostatic field applied to the film surface has been found to increase the activation energy of flux creep in relatively low magnetic fields. The upper critical magnetic field determincd from resistive measurements increases in the vicinity of superconducting transition temperature under the influence of the electrostatic field.
\end{abstract}

\section{INTRODUCTION}

It is well known that critical currents of the Bi-based high- $\mathrm{T}_{\mathrm{C}}$ superconductors are low because of weak pinning resulting in enhanced dissipation originated from magnetic flux motion. Therefore, use of this material in high transport current applications or in high external magnetic fields is limited. But in the case of applications to low temperature electronics, the magnetic fields (an ambient field and so-called self-field due to the transport current through the superconductor) are usually weak, typically less than a few hundred Oersted, so dissipation behaviour and the pinning potential in relatively weak magnetic fields are of interest.

Studies of behaviour of superconductors in a strong electrostatic field are important from a standpoint of the search for potential application of these materials in voltage-controlled transistor-like superconducting devices. Recent experiments have shown that both the critical temperature of superconducting transition $T_{c}$ and the critical current density $J_{c}$ of high- $T_{c}$ superconducting thin films can be modulated by an electrostatic field applied to the sample surface $[1-3]$. The negative electrostatic field applied to the surface of high- $T_{c}$ samples was found to change the upper critical magnetic field $\mathrm{H}_{\mathrm{c} 2}$ [4] and fluctuation enhanced excess conductivity above $\mathrm{T}_{\mathrm{c}}[5,6]$.

On the other hand, application of the electrostatic field enables to modulate concentration of mobile charge carriers near the sample surface. The charging state of the near surface pinning centers determines their electrophysical properties, for example, activation energy $U_{0}$ associated with the depth of the potential well, so measurements of dissipation near the zero resistance state provide us with information about the physical nature of the pinning centers and about mechanisms of interaction between the pinning center and the flux line.

In this paper we describe measurements of dissipation near the zero-resistance state and of temperature dependences of the upper critical magnetic field in the polycrystalline $(\mathrm{Bi}, \mathrm{Pb})_{2} \mathrm{Sr}_{2} \mathrm{Ca}_{2} \mathrm{Cu}_{3} \mathrm{O}_{\mathrm{x}}$ thin film under the strong electrostatic field. We have measured temperature dependences of the pinning potential $U_{0}$ in the magnetic fields ranging from 10 to $5000 \mathrm{Oe}$ and determined the electric field effect on $\mathrm{U}_{0}$. 


\section{EXPERIMENTAL}

A polycrystalline $(\mathrm{Bi}, \mathrm{Pb})_{2} \mathrm{Sr}_{2} \mathrm{Ca}_{2} \mathrm{Cu}_{3} \mathrm{O}_{\mathrm{x}}$ film, $0.78 \mu \mathrm{m}$ thick, was deposited onto a $\mathrm{MgO}$ substrate. Resistive measurements were made by the standard DC four probe method. To apply an electrostatic field to the film surface the structure "metal-insulator-superconductor" was used. The insulating layer was a $15 \mu \mathrm{m}$ thick mylar film pressed to the sample surface by the polished copper plate used as a field (or gate) electrode. The sign of the voltage bias applied to the gate electrode was chosen to be negative in order to induce near the surface a layer enriched with holes which are majority charge carriers in high- $T_{c}$ superconductors of this type. Application of $-700 \mathrm{~V}$ gate bias resulted in the electric field strength of about $5 \times 10^{5} \mathrm{~V} / \mathrm{cm}$ near the surface.

Resistive measurements in the vicinity of the zero-resistance state have shown that dissipation in this temperature region is mainly determined by the thermally activated motion of the magnetic flux. According to the flux creep theory [7] the resistive "tails" of the superconducting transition obey the Arrhenius law: $\mathrm{R}=\mathrm{R}_{0} \times \exp \left(\mathrm{U}_{0} / \mathrm{kT}\right)$, where $\mathrm{U}_{0}$ is the activation energy of the flux creep. From the slopes of the Arrhenius plots $\left(\ln \left(R / R_{0}\right)\right.$ vs. $\left.1 / T\right)$ the vortex activation energy $U_{0}$, which is related to the potential well depth of the individual pinning center, can be determined.

The upper critical magnetic field was determined from the resistance-temperature curves using a criterion of $0.05 \times R_{N}$, where $R_{N}$-linear approximation of the normal-state resistance. The magnetic field was normal to the sample surface and to the transport current direction. Electrostatic and magnetic fields were applied in the resistive state and maintained constant during the measuring cycle.

\section{RESULTS AND DISCUSSION}

\subsection{Temperature dependences of the pinning potential}

Critical temperature $\mathrm{T}_{c}$ of the investigated $(\mathrm{Bi}, \mathrm{Pb})_{2} \mathrm{Sr}_{2} \mathrm{Ca}_{2} \mathrm{Cu}_{3} \mathrm{O}_{\mathrm{X}}$ film determined under a criterion of maximum derivative of $\mathrm{R}(\mathrm{T})$ dependence was $107.4 \mathrm{~K}$.

Figure 1 shows the activation energy $\mathrm{U}_{0}$ as a function of temperature for different external magnetic fields. It can be seen in the figure that there are three different shapes of $U_{0}(T)$ dependences in different ranges of the magnetic field. When the magnetic field is low (less than 20 $\mathrm{Oe}), \mathrm{U}_{0}(\mathrm{~T})$ appears as a curve with a maximum. In intermediate fields (50-100 Oe in our case) activation energy increases monotonically with temperature and in "high" fields (more than 200 $\mathrm{Oe}$ ) it is approximately constant in the wide temperature interval. We use here the definition "high" for the third range of magnetic fields though they are rather low in the conventionally adopted sense.

Curves with a maximum may result from an interplay between two competing mechanisms. The pinning theory based on the model assumption of pinning of an isolated vortex (quantum flux line) at the individual center predicts power law dependence of the activation energy on reduced temperature of the form: $\mathrm{U}_{0}(\mathrm{~T})=\mathrm{U}\left(1-\mathrm{T} / \mathrm{T}_{\mathrm{C}}\right)^{\mathfrak{q}}$ with $\mathrm{q}>0$ [8]. According to this relation the activation energy should decrease with $T$ coming to zero at $T_{c}$. Increase of $U_{0}$ with $T$ can be explained by at least three ways. First, if there is a distribution of pinning energies, some of which have very low values, then only the sites with appropriate well-depths are sampled at a given temperature [9]. It should be mentioned that such an explanation was suggested for $U_{0}$ determined from the magnetization relaxation measurements. Second possibility appears if the assumption of a linear relation between the Lorentz force and activation energy is incorrect [10]. It has been shown [11] that the apparent activation energy $\mathrm{U}_{0}$ obtained from flux creep data using the above assumption may go to zero as the temperature decreases to zero even when the true pinning-well depth $U_{p}$ is independent of $T$. Third reason may come from a fact that as the temperature increases 
small parts of the material with lower $\mathrm{T}_{\mathrm{C}}$ (for example, oxygen deficient regions) transform to the normal phase and serve as additional pinning centers [12].

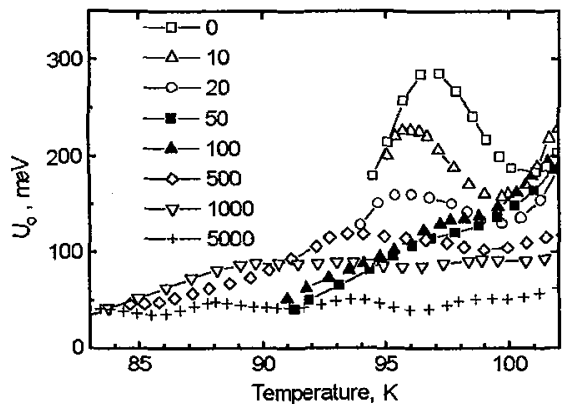

Figure 1: Temperature dependences of pinning energy for different magnetic fields.

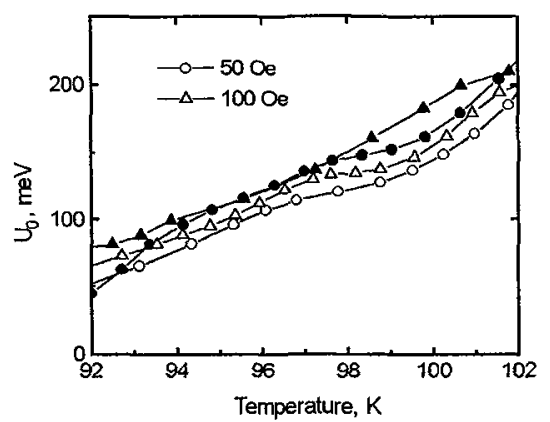

Figure 3: Pinning energies at intermediate magnetic fields for zero (open) and negative (solid) voltage.

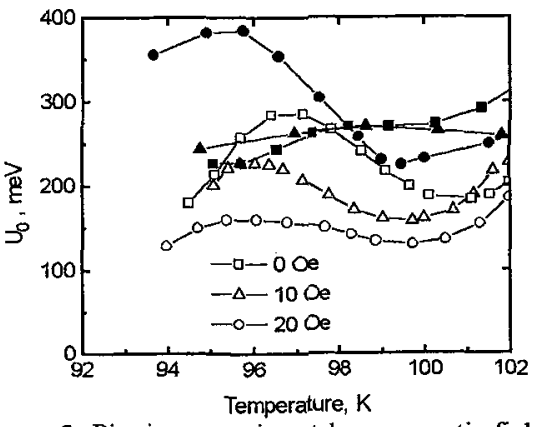

Figure 2: Pinning energies at low magnetic fields for zero (open symbols) and negative (solid symbols) voltage applied to the surface.

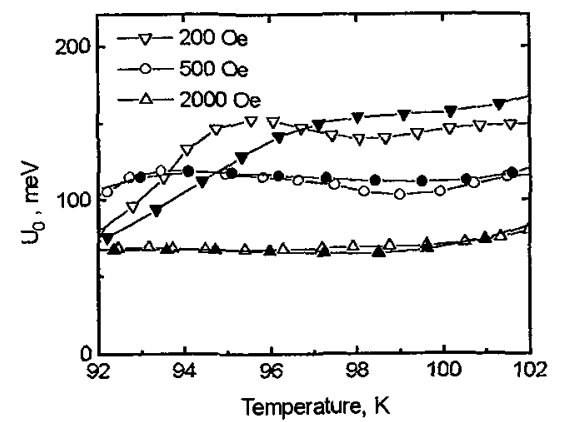

Figure 4: Pinning energies at high magnetic fields for zero (open) and negative (solid) gate voltage.

Differences in the behaviour of $\mathrm{U}_{0}$ in various magnetic fields can be also attributed to features of flux penetration into a type II superconductor. It has been shown [13] that in low magnetic fields flux penetrates only into a thin surface region of the sample and dissipation is determined by pinning in this thin layer. At greater magnetic fields the flux profile across the sample becomes nearly constant and relative contribution of bulk pinning increases. Considering our three magnetic field regions we can assume that in the low field regime magnetic flux lines penetrate into the thin surface layer, in intermediate fields they penetrate the sample bulk as isolated noninteracting fluxons and at higher fields the classic Abrikosov flux line lattice is formed. The fact that at high fields the activation energy is approximately constant in the wide temperature range reflects the process of Abrikosov lattice melting due to thermally activated flux creep.

\subsection{Effect of the electrostatic field on $U_{0}$}

The effect of the electrostatic field applied to the surface of the superconducting film on the $U_{0}(T)$ dependences is shown in figures 2,3 and 4 . It can be seen in the figures that the negative electric field increases the activation energy. As was expected, the most prominent effect is observed in low magnetic fields (Fig.2) when contribution of surface pinning centers is large. In intermediate magnetic fields (Fig.3) the increase in $\mathrm{U}_{0}$ also takes place, but the magnitude of the effect becomes lower due to increase of bulk contribution into dissipation. In high magnetic fields (Fig.4), when 
dissipation is mainly determined by bulk pinning, application of the electric field has no influence on $\mathrm{U}_{0}$.

Since all electric field effects are known to be surface related, just surface pinning centers should be responsible for the observed changes in $U_{0}$. The mechanism of the electric field effects in high$\mathrm{T}_{\mathrm{c}}$ superconductors is still being debated. Electric field induced enhancement of the critical current density in Y-based superconductors was associated with either modulation of the well-depth of the individual pinning site $[14,15]$ or better intergrain coupling [2]. Our present results indicate that in $\mathrm{Bi}$ - based superconductors the first mechanism seems to be dominant.

As has been mentioned above, in low magnetic fields the penetration depth of fluxons is much less than the film thickness. It means that in the near-surface layer direction of flux lines does not coincide with that of the external field, so that fluxons may be oriented not perpendicular to the film surface (which is parallel to the CuO planes) but at any angle or even parallel to it. It is well known, that in extremely anisotropic $\mathrm{Bi}$ - superconductors fluxons can be pinned at the discreteness of the crystal lattice if their cores lie between superconducting $\mathrm{CuO}$ planes [16] (so called intrinsic pinning). By inducing excess charge in the $\mathrm{CuO}$ planes near the surface we increase a potential relief and intrinsic pinning becomes stronger.

The electric field effect on flux pinning can be explained taking into account "pancake vortex" model for highly anisotropic superconductors [17]. According to this picture, due to weak interlayer coupling, small segments of a vortex can be displaced independently, allowing them to move by thermal activation at relatively low temperature. The dimension of the movable "pancake" stack along c-axe (perpendicular to the $\mathrm{CuO}$ planes) depends on the interlayer Josephson coupling between planes. Increase of charge carrier concentration leads to better interlayer coupling, the number of "pancakes" in the stack increases and as a result a fluxon becomes less mobile.

The above explanations agree with supposition of Mannhart et al. [14] that the field-induced modulation of the carrier concentration changes the depth of the individual pinning site, so changing the critical current density $J_{c}$ in the high- $T_{c}$ thin film.

\subsection{Dependence of activation energy on the magnetic field}

Figure 5 shows another "cross-section" of $U_{0}(T, H)$ dependences: $U_{0}$ vs. $H$ curves for different temperatures, 99 and $101 \mathrm{~K}$. The effect of the negative electrostatic field is also demonstrated in the figure.

The zero voltage curves are similar to the $J_{c}(H)$ dependences both reported by Ekin et al. for $\mathrm{Bi}$ - based superconductor at $76 \mathrm{~K}$ [18] and found in our previous measurements for the temperature range from 65 to $90 \mathrm{~K} \mathrm{[3].} \mathrm{In} \mathrm{[18]} \mathrm{first} \mathrm{drop} \mathrm{in} \mathrm{J}_{\mathrm{C}}(\mathrm{H})$ curve is attributed to Josephson weak link limitation of the critical current density, the plateau region was associated with existence of remnant percolation paths and second drop is related to the flux creep regime. It should be noted that experimental data referred in [18] shows that $J_{C}$ in the second region is not constant but rises with increase of the magnetic field, which can not be explained in frames of the percolation path model. Based on our measurements we can suppose that the plateau region in $J_{c}(H)$ dependences is directly related to the same behaviour of $U_{0}(H)$ and the percolation model is not necessary for explanation of $\mathrm{J}_{\mathrm{c}}(\mathrm{H})$ data.

The physical mechanism of the observed rise in $U_{0}$ with the magnetic field can be the same as described above. As the magnetic field increases, the regions of superconductor with lower value of upper critical magnetic field $\mathrm{H}_{\mathrm{c} 2}$ become normal and produce additional pinning sites. The electrostatic field has been found to increase the activation energy of flux pinning at magnetic fields less than $200 \mathrm{Oe}$. 


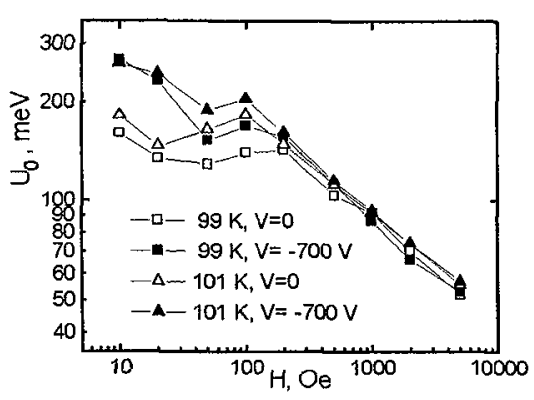

Figure 5: Dependences of the activation energy on the magnetic field for temperatures 99 and $101 \mathrm{~K}$. Solid symbols correspond to application of the negative electric field to the film surface.

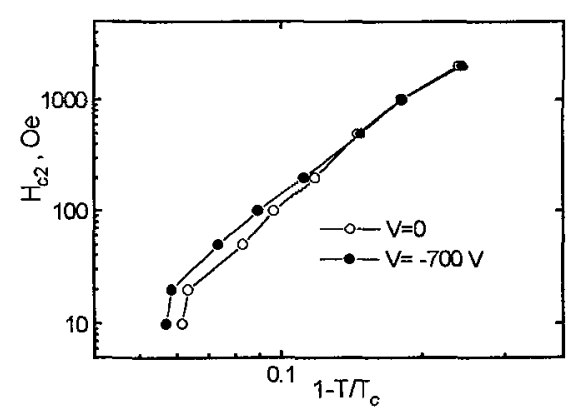

Figure 6: Dependences of the upper critical magnetic field for zero (open) and negative (solid) voltage at the sample surface.

\subsection{Upper critical magnetic field}

Figure 6 shows dependences of the upper critical magnetic field $\mathrm{H}_{\mathrm{c} 2}$ on the reduced temperature for zero and negative voltage at the sample surface. The effect of the electrostatic field have been found to occur in the higher temperature region, whereas at lower temperatures and at higher $\mathrm{H}_{\mathrm{c} 2}$ values, correspondingly, the electric field effect disappeared. This result can be attributed to the fact that in the higher magnetic fields the penetration depth of the magnetic field into the sample becomes more than that of the electric field, and dissipation is mainly determined by bulk pinning centers.

Enhancement of $\mathrm{H}_{\mathrm{c} 2}$ under the negative electrostatic field was observed in high- $\mathrm{T}_{\mathrm{c}}$ ceramic YPrBaCuO samples [4]. Comparing those data with our present results one can see that in $\mathrm{Bi}$ - film there is no region in the $\mathrm{H}_{\mathrm{c} 2}$ vs. $\mathrm{T}$ dependence related to intergrain weak links. Therefore, we must draw the conclusion than intergrain coupling is much better in $\mathrm{Bi}$ - based superconductors than in Y- based ceramics.

\section{Conclusions}

Thus, we have shown that there are different ranges of the external magnetic field, in which the dissipation mechanisms may be essentially different. At low magnetic fields (less than $200 \mathrm{Oe}$ ) the transport properties of Bi-based superconducting thin film can be modulated by the external electrostatic field applied to the film surface. The activation energy of flux pinning can be increased by a factor of two by application of the negative voltage at the sample surface. In higher magnetic fields the electric field effect disappears due to the increase of bulk contribution to dissipation.

Measured $\mathrm{U}_{0}(\mathrm{H})$ dependences are in qualitative agreement with $\mathrm{J}_{\mathrm{C}}(\mathrm{H})$ data. This makes possible to explain nonmonotonous $\mathrm{J}_{\mathrm{c}}$ behaviour in the magnetic field.

The electric field effect on the vortex dynamics in thin high- $\mathrm{T}_{\mathrm{c}}$ films can be used in electronics for fabrication of the vortex-flow transistor controlled by an external voltage bias.

\section{Akcnowledgements}

The research described in this publication was made possible in part by Grant No. U5U200 from the International Science Foundation and State of Ukraine. 


\section{References}

[1] Mannhart J., Bednorz J.G., Müller K.A., Schlom D.G., Z. Phys. B - Condensed Matter. 83 (1991) 307-311.

[2] Gomeniuk Y.V., Lozovski V.Z., Lysenko V.S., Tyagulski I.P., Physica C 214 (1993) 127132.

[3] Lysenko V.S., Gomeniuk Y.V., Lozovski V.Z., Tyagulski I.P., Osiyuk I.N., Varyukhin V.N., "Dissipation behaviour and critical currents under the strong electrostatic field in the $\mathrm{BiPbSrCaCuO}$ thin film", Symposium on Low Temperature Electronics and High Temperature Superconductivity, Reno, Nevada, 21-26 May 1995, C.L.Claeys, S.I.Raider, R.K.Kirschman, W.D.Brown Eds. (The Electrochem. Soc. Inc., Pennington, 1995) pp.148-154.

[4] Gomeniuk Y.V., Lozovski V.Z., Lysenko V.S., Tyagulski I.P., Variukhin V.N., Phys. Status Solidi(a) 132 (1992) 155-161.

[5] Gomeniuk Y.V., Lozovski V.Z., Lysenko V.S., Tyagulski I.P., Solid State Comm., 85 (1993) 643-646.

[6] Lysenko V.S., Gomeniuk Y.V., Lozovski V.Z., Tyagulski I.P., Varyukhin V.N., Physica C 235-240 (1994) 2631-2632.

[7] Anderson P.W. and Kim Y.B., Rev. Mod. Phys. 36 (1964) 39-43.

[8] Yeshurun Y. and Malozemoff A.P., Phys.Rev.Lett. 60 (1988) 2202-2205.

[9] Hagen C.W. and Griessen R., Phys.Rev.Lett. 62 (1989) 2857-2860.

[10] Beasley M.R., Labusch R., Webb W.W., Phys.Rev. 181 (1969) 682-700.

[11] Welch D.O., IEEE Trans. on Magnetics 27 (1991) 1133-1138.

[12] Nishi Y., Nozaki K., Ichimura S., Journ. of Applied Phys. 71 (1992) 5569-5571.

[13] Cave R.J., Critchlow P.R., IEEE Trans. on Magnetics 27 (1991) 1379-1382.

[14] Mannhart J., Schlom D.G., Bednorz J.G., Müller K.A., Phys.Rev.Lett. 67 (1991) 2099-2101.

[15] Walkenhorst A., Doughty C., Xi X.X., Li Q., Lobb C.J., Mao S.N., Venkatesan T., Phys.Rev.Lett. 69 (1992) 2709-2712.

[16] Maley M.P., Journ. of Applied Phys. 70, Pt.2 (1991) 6189-6193.

[17] Clem J.R., Physica C 153-155 (1988) 50-55.

[18] Ekin J.V., Larson T.M., Hermann A.M., Sheng Z.Z., Togano K., Kumakura H., Physica C 160 (1989) 489-496. 